\title{
¿Hacia un nuevo ciclo extractivista? Revisitando la relación entre comunidades, conflictos e industrias extractivas
}

\author{
Felipe Irarrázaval',2 (0), Ignacio Rojas Rubio ${ }^{3}$, \\ Rodrigo Hidalgo Dattwyller ${ }^{4}$ (1), Sasha Rincón Quiroz ${ }^{5}$ (1), \\ Juan Camilo Álvarez ${ }^{6}$ (1), Voltaire Alvarado Peterson ${ }^{7}$, \\ Xenia Fuster-Farfán ${ }^{8}$ (), Oliver Meseguer Ruiz ${ }^{9}$ (i) y \\ Fabian Lizana Vásquezio (i)
}

\begin{abstract}
El último superciclo de los commodities, el cual se puede situar desde inicios del 2000 hasta el 2015, significó un aumento sin precedentes en el desarrollo de proyectos para la extracción de recursos naturales a gran escala en América Latina. Tras un relativo declive del sector en los últimos años, hay dos aspectos coyunturales que marcarán el rumbo de una nueva etapa de auge de las industrias extractivas. En primer lugar, el estancamiento económico asociado a las medidas sanitarias en torno a la pandemia del COVID 19 comienza a menguar, y se avizoran distintos planes de reactivación económica en la región. Dada la especialización de la región en la división internacional del trabajo, esta reactivación contará con un componente protagónico del sector extractivo. La pregunta clave es en qué medida los gobiernos de la región facilitarán la aprobación de proyectos extractivos, y cómo esta facilitación podría menoscabar las débiles instancias de consulta y participación por parte de las comunidades locales. En segundo lugar, la contundencia del Sexto Informe del IPCC (2021) pondrá mayor exigencia sobre transiciones energéticas hacia economías bajas en carbono a nivel global. Este desafío global requiere del desarrollo masivo de nuevas tecnologías para generación energética, como aspas eólicas o paneles solares, así como también vehículos eléctricos. Todas estas tecnologías requieren una cantidad significativa de minerales, como por ejemplo cobre, litio y cobalto. Por este motivo, la transición energética conlleva una expansión del sector extractivo, especialmente minero, en América Latina.
\end{abstract}

\footnotetext{
El autor agradece al Centro de Estudios de Conflicto y Cohesión Social (COES) ANID/FONDAP/15130009.

Editor Invitado, Revista de Geografía Norte Grande, Instituto de Estudios Urbanos y Territoriales, Pontificia Universidad Católica de Chile Correo electrónico: firarrazava@uc.cl

Universidad de Playa Ancha, Instituto de Geografía, Pontificia Universidad Católica de Chile. Correo electrónico: Ifrojas1@uc.cl

Instituto de Geografía, Pontificia Universidad Católica de Chile. Correo electrónico: rhidalgd@uc.cl

Instituto de Geografía, Pontificia Universidad Católica de Chile. Correo electrónico: sjrincon@uc.cl

Instituto de Geografía, Pontificia Universidad Católica de Chile. Correo electrónico: jcalvarez2@uc.cl

Departamento de Geografía, Universidad de Concepción. Correo electrónico: voalvarado@udec.cl

Revista de Geografía Norte Grande, Pontificia Universidad Católica de Chile. Correo electrónico: xenia.fuster@gmail.com

Departamento de Ciencias Históricas y Geográficas, Universidad de Tarapacá. Correo electrónico: omeseguer@academicos.uta.cl

Instituto de Geografía, Pontificia Universidad Católica de Chile. Correo electrónico: fslizana@uc.cl
} 
Estos dos aspectos permiten presagiar una nueva etapa de auge para el sector extractivo en la región. En ese sentido, resulta clave revisitar las múltiples contradicciones asociadas a la estrategia de acumulación basada en la explotación de recursos naturales a gran escala impulsada por los gobiernos latinoamericanos, y proyectar su manifestación en este nuevo ciclo. Para ese propósito, no sólo es relevante proyectar la activa agenda de investigación respecto a cómo el extractivismo ha sido disputado desde la escala local (Bebbington y Bury 2014; Bergamini y Pérez 2015; Gudynas 2009; Svampa 2012), sino también las contribuciones que han invitado a reflexionar respecto a las dinámicas actuales de los conflictos socio-territoriales y su efecto agregado sobre las instituciones (ver por ejemplo Dargent et al., 2017; Delamaza, 2019; Farthing \& Fabricant, 2018; Haarstad, 2012; Silva et al., 2018). En otras palabras, también es necesario examinar cómo los cambios políticos recientes han modificado las formas de relación entre industrias, Estado y comunidades locales.

Por un lado, Mantovani \& Svampa (2019) plantean que es necesario identificar las dinámicas actuales que emplean los regímenes políticos de la región y que intensifican la apropiación de la naturaleza como, por ejemplo, estados de urgencia y formas de violencia más radical sobre los activistas ambientales. Por otro lado, hay una serie de condiciones políticas que se han consolidado o emergido, y que han posibilitado el escalamiento de los conflictos socio-territoriales más allá de lo local. Por ejemplo, el reconocimiento de convenios internacionales (EITI, OIT 169 y potencialmente Escazú), la emergencia de formas no tradicionales de ciudadanía (Delamaza 2019; Rich, Mayka y Montero 2019) o reformas de descentralización que han posibilitado nuevos bloques o actores políticos subnacionales (Irarrazaval 2020; Riofrancos 2017). Si bien para los actores locales sigue siendo difícil trascender espacios locales, estas condiciones posibilitaron la emergencia y el posicionamiento político de diferentes grupos que han buscado influir en la gobernanza de los recursos naturales (Haarstad 2014; Riofrancos 2017). Adicionalmente, se puede mencionar que las industrias extractivas también han modificado su manera de relacionarse con el territorio para obtener licencia social para operar (Amengual 2018; Leiva 2019; Moffat y Zhang 2014; Ocampo-Melgar, Sagaris y Gironás 2019).

¿Qué significan estos cambios para las contradicciones entre los modelos de desarrollo basados en la extracción de recursos y los conflictos socio-territoriales? Si bien estas dinámicas no resuelven las contradicciones territoriales del modelo, la globalidad de estos cambios insta a examinar si las dinámicas actuales están produciendo una configuración territorial diferente. En ese sentido, es necesario realizar una reflexión profunda que permita identificar la contingente articulación entre las industrias extractivas, los Estados y los proyectos locales de gobernanza sobre los recursos naturales en el escenario actual. En ese contexto, este número especial denominado"'Extractivismos'y conflictos socio-territoriales en Latinoamérica: Enfoques posibles y líneas de investigación emergentes" busca revisitar las tensiones y contradicciones asociadas al 'extractivismo' en América Latina, y particularmente examinar campos emergentes en los conflictos socio-territoriales asociados a la apropiación de la naturaleza, así como ampliar la gama de posibles enfoques para rediscutir los conceptos centrales de conflicto, territorio, extractivismo y naturaleza.

Los trabajos de este número provienen de distintos lugares de América Latina, y contribuyen a la urgencia de revisitar y actualizar la agenda de investigación desde distintos ángulos. Astrid Ulloa (2021) profundiza explícitamente en los procesos de cambio planteados en esta introducción, y da cuenta de cómo se manifiestan en la Guajira, Colombia, distintas formas de extractivis- 
mo. Dentro de esta multiplicidad de formas, emergen las reconfiguraciones extractivistas disfrazadas en discursos de energías verdes y azules, las que son una nueva forma de reconfiguración de desigualdades étnicas, de género y territoriales en el marco del extractivismo. En ese sentido, presenta la urgencia de replantear las relaciones socioambientales para promover transformaciones ambientales radicales. La contribución de Ximena Cuadra Montoya (2021) también avanza en esa dirección al abordar un caso de energías renovables no convencionales (las cuales son el puntal de la transición global hacia economías bajas en carbono) en territorio mapuche en Chile. Asimismo, su trabajo profundiza en cómo la implementación de este tipo de proyectos en territorio indígena construye un multiculturalismo neoliberal extractivo, cimentado sobre procesos de consulta que limitan las posibilidades de inclusión y exclusión de sujetos.

Maillet et al. (2021) presentan una revisión sistemática de la literatura sobre extractivismo, conflicto y territorio en Chile. Se plantea que esta agenda es muy convergente en términos conceptuales y metodológicos, y se invita a complejizar el contenido conceptual de la popular triada para estudiar la relación entre comunidades e industrias extractivas: conflicto, territorio y extractivismo. Eso es, en efecto, lo que hacen Bayón et al., (2021), quienes tensionan la forma en que la Ecología política ha abordado los conflictos en torno al extractivismo, mediante un enfoque que dialoga entre la Ecología política y las Geografías críticas de la movilidad. Con estudios de caso en la Amazonía ecuatoriana, dan cuenta de la urgencia de reconocer explícitamente las trayectorias de vida y prácticas de movilidad de los sujetos al momento de examinar los despojos extractivistas. Escalona y Barton (2021) también apuntan en la dirección de incorporar de mejor manera los procesos históricos en el análisis de los conflictos territoriales actuales, particularmente del caso del Wallmapu en Chile. Estos autores proponen una Ecología política histórica para comprender la construcción de paisaje de poder como punto de entrada para examinar las configuraciones socio-ecológicas sobre las que acontecen. Thomas (2021) aporta un enfoque de género para examinar las relaciones entre mujeres y minería en Choapa alto, Chile. Si bien converge con las contribuciones que entienden a la minería como un territorio masculinizado, aborda específicamente cómo los esfuerzos de las empresas por incorporar a mujeres en la fuerza de trabajo, han generado escenarios contradictorios y complejos, donde roles e identidades de género tradicionales se tienden a reforzar.

\section{El presente número}

El Número 80 se presenta como un hito importante en los 47 años de publicación continua de la Revista de Geografía Norte Grande (RGNG). Junto a la publicación de este número semi-temático, con seis artículos, se presentan 12 trabajos que abordan una diversidad temática de interés para la Geografía.

Además, por medio de un esfuerzo colectivo, se ha logrado poner a disposición, en línea, el archivo histórico de la revista, desde el Número 1 publicado en el año 1974, hasta el presentado en esta editorial. Contar con este repositorio es una contribución a la Geografía del país y del continente, pensando en que los principales usuarios son estudiantes de distintos niveles y que usan estos artículos en sus trabajos de investigación. Esto queda refrendado en las casi 8500 citas que la RGNG ostenta en su perfil de Google Académico. 
Junto a lo anterior, la revista está viviendo un proceso de actualización de los procesos de gestión editorial y de las normas de publicación. Esto significa incorporar nuevos softwares que permiten el reconocimiento de trabajos originales, que cumplen con los estándares éticos internacionales en la citación y uso de fuentes. Asimismo, hemos incorporado nuestra revista a nuevas plataformas digitales, como Google Scholar, que permite una mayor visualización, difusión y divulgación de los trabajos que se encuentran en línea. Esta acción se fundamenta en el principio ético de que el conocimiento es de acceso abierto y libre.

El mantener la publicación de trabajos en castellano y portugués como idiomas principales de publicación ha permitido un alto flujo de trabajos que se gestionan por la revista. Destacamos la calidad de los trabajos publicados de estudiantes de magíster y doctorado, investigadoras e investigadores jóvenes de diferentes rincones de América Latina y el gran interés de lectura que posee nuestra publicación por estudiantes de pregrado, visualizado por medio de la descarga de archivos.

Otro hito importante que hemos vivido el último año es la migración y actualización de nuestra plataforma de gestión de artículos OJS, la cual se ha integrado a un sistema común de revistas de la Pontificia Universidad Católica de Chile, lo cual también permite una mayor visualización de la Revista de Geografía Norte Grande.

El número semi-temático que lleva por nombre ¿Hacia un nuevo ciclo extractivista? Revisitando la relación entre comunidades, conflictos e industrias extractivas expresa el interés por los conflictos espaciales, territoriales y/o medio ambientales en diferentes ciencias, lo cual se visualiza en el interés temático de los trabajos publicados en los últimos 10 años. Los artículos que son parte del misceláneo de este número expresan la alta productividad en estudios de caso sobre conflictos ambientales, planificación y normatividad urbana y ambiental, efectos de los sistemas productivos sobre los ambientes naturales, políticas de conservación de espacios naturales, extractivismo urbano y desigualdad socio espacial.

El trabajo de Constanza Tommei y Patricia López, denominado Herramientas para proyectar el territorio en el siglo XXI en América Latina, aporta con una aproximación a los conceptos más utilizados para planificar un territorio: Urbanismo, Planificación, Proyecto Urbano, Ordenamiento Territorial, Código de Planeamiento Urbano y Planificación Estratégica. Por su parte, Geomorfositios en el Geoparque Mundial Unesco de El Hierro (Islas Canarias, España) para fomentar el geoturismo en espacios volcánicos, de los autores Javier Dóniz, Rafael Becerra-Ramírez y la autora Esther Beltrán, identifica, inventaría, selecciona y valora veinte geomorfositios volcánicos destacados que tienen interés geoturístico para fomentar su visita y que diversificar la oferta de ocio en las islas Canarias.

Luego, continúa el artículo trabajado por Ignacio Trucco y Victor Ramiro que han denominado Proximidad, territorio e innovación. Una aproximación crítica desde categorías ausentes: escalaridad, estructuración social y periferia. Pone el foco en las relaciones entre proximidad, territorio e innovación. El debate se estructuró en torno a dos preguntas subyacentes: ¿qué liga a la sociedad con el espacio geográfico? y ¿cuáles son las razones que definen su desempeño económico? En el artículo de Klaus Hennicke y Luis Davinson se aborda una problemática ambiental asociada al legado contaminante de relaves mineros que operaron en el territorio austral de Chile, 
en un pasado reciente, y que denominaron Conflictividad ambiental en botaderos mineros de comunidades del Lago General Carrera. Retrospectiva desde actores locales de Aysén, Chile.

La investigadora Karen Andersen y el investigador Cristóbal Balbontín de la Universidad Austral de Chile, nos presentan la investigación denominada La planificación del borde costero chileno. Una normativa deficiente, que, a través de un análisis jurídico, plantea una crítica en relación a la participación ciudadana y al valor del paisaje y medioambiente en la planificación del territorio costero. El siguiente trabajo también es de la Región de Los Ríos, se le ha asignado el título Repercusiones de las macro-políticas de industrialización por sustitución de importaciones y de innovación en la dinámica urbana de los Barrios Bajos, Valdivia, de las autoras Laura Rodríguez, Tirza Barría y el autor Robinson Silva, explora las repercusiones de las macro-políticas de sustitución de importaciones y de innovación en la espacialidad de los Barrios Bajos, Valdivia y su efecto transformador en el corto y largo plazo.

Análisis de conectividad del paisaje para tres especies de mesodepredadores altoandinos como herramienta para su conservación, es como han denominado Juan Jiménez-Ramírez y Hugo López-Arévalo a su investigación que evaluó la distribución potencial del cusumbo, zorro y oncilla en el norte de la Sabana de Bogotá, región de Colombia, que presenta altas transformaciones por actividades antrópicas. También pueden encontrar en este número el trabajo de Eugenio Cejudo, Gabriel da Silva y Francisco Navarro llamado Resultados de la implementación del desarrollo rural territorial. Lecciones del enfoque LEADER en España versus el programa Territorios de la Ciudadanía en Brasil, sistematiza los logros alcanzados, en Europa a través del enfoque $L E A D E R$ como Brasil con el programa Territorios de la Ciudadanía, en relación con los principios que sustentan este nuevo enfoque del desarrollo rural territorial.

El siguiente trabajo vinculado a los estudios urbanos, denominado iAl mirarte de Playa Ancha, lindo puerto! Edificación en altura, renta de suelo y extractivismo urbano en Valparaíso (1991-2017) de Carlos Vergara-Constela y Nelson Carroza, trata sobre la edificación en altura en la comuna de Valparaíso y se muestra la superficie construida, su cantidad, localización y las rentas obtenidas de acuerdo con las temporalidades y los espacios de la ciudad. En el mismo campo de estudio es el trabajo que se desarrolla por Julián Grijalba, que se titula Spatiotemporal variability of urban soundscape: a cross-continental comparison between Venice (Italy) and Popayán (Colombia), y que examina el paisaje sonoro de los cascos históricos de Venecia (Italia) y Popayán (Colombia). En ese sentido, se realizaron cartografías de los factores físico y perceptual del paisaje sonoro urbano con el propósito de garantizar su eficaz evaluación.

Los últimos dos trabajos del presente número hacen referencia a la "diferenciación" desde dos campos de estudios. El primero, se concentra en el estudio de Las desigualdades en el acceso al agua en ciudades latinoamericanas de rápido crecimiento: el caso de Arequipa, Perú, a través de encuestas en centro y periferia de la ciudad, y con el análisis de datos secundarios, se contrasta la prestación de servicios mediante la caracterización de la accesibilidad, la disponibilidad, la calidad del servicio, el precio y la preferencia del modelo de gestión (pública o privada), y que fue trabajado por Luis Zapana, Hug March y David Sauri. Y, Diferencias en el ensamble de micromamíferos entre áreas con distinto grado de protección del sitio Prioritario los Molles-Pichidangui de Cristián Larraguibel, Dusan Boric, Juan L. Celis, Rodrigo Figueroa y Fernando Torres, describen el ensamble de micromamíferos del Bioparque Puquén y alrededores comparando la 
riqueza de especies, abundancia relativa e índice de diversidad de Margalef al interior y exterior del parque. Mediante el índice de similitud de Morisita-Horn y un análisis de agrupamientos se comparó el ensamble de micromamíferos entre puntos de muestreos categorizados en distintos biotopos.

\section{Referencias}

AMENGUAL, M. Buying stability: The distributive outcomes of private politics in the Bolivian mining industry. World Development, 2018, vol. 104, pp. 31-45. ISSN 0305750X. DOI 10.1016/j.worlddev.2017.11.008.

BEBBINGTON, A. y BURY, J. Subterranean Struggles: New Dynamics of Mining, Oil, and Gas in Latin America. Reprint edition. S.I.: University of Texas Press, 2014.

BERGAMINI, K. y PÉREZ, C. Fiscalización y cumplimiento ambiental en Chile: principales avances, desafíos y tareas pendientes. EURE (Santiago), 2015, vol. 41, no. 124, pp. 267-277. ISSN 0250-7161. DOI 10.4067/SO250-71612015000400013.

DARGENT, E., ORIHUELA, J.C., PAREDES, M. y ULFE, M.E., 2017. Cycle of Abundance and Institutional Pathways. Resource Booms and Institutional Pathways [en línea]. S.I.: Palgrave Macmillan, Cham, Latin American Political Economy,, 2015, pp. 1-40.

DELAMAZA, G. Consecuencias políticas de los conflictos socio-territoriales. Hacia una conceptualización pertinente. Revista Austral de Ciencias Sociales, 2019, no. 37, pp. 139-160. ISSN 0718-1795. DOI 10.4206/rev.austral.cienc.soc.2019.n37-08.

FARTHING, L. y FABRICANT, N. Open Veins Revisited: Charting the Social, Economic, and Political Contours of the New Extractivism in Latin America. Latin American Perspectives, 2018, vol. 45, no. 5, pp. 4-17. ISSN 0094-582X. DOI 10.1177/0094582X18785882.

GUDYNAS, E. Diez tesis urgentes sobre el nuevo extractivismo. Contextos y demandas bajo el progresismo sudamericano actual. Extractivismo, política y Sociedad. Quito: CAAP-CLAES, , 2009.

HAARSTAD, H. New Political Spaces in Latin American Natural Resource Governance. 2012 edition. New York: Palgrave Macmillan. ISBN 978-0-230-34070-1.

HAARSTAD, H. Cross-scalar Dynamics of the Resource Curse: Constraints on Local Participation in the Bolivian Gas Sector. The Journal of Development Studies, , 2014, vol. 50, no. 7, pp. 977-990. ISSN 0022-0388. DOI 10.1080/00220388.2014.909026.

IPCC Climate Change 2021: The Physical Science Basis. Contribution of Working Group I to the Sixth Assessment Report of the Intergovernmental Panel on Climate Change. . S.I.: Cambridge University Press, 2021. 
IRARRAZAVAL, F. Contesting uneven development: The political geography of natural gas rents in Peru and Bolivia. Political Geography, 2020, vol. 79, pp. 102161. ISSN 0962-6298. DOI 10.1016/j. polgeo.2020.102161.

LEIVA, F. Economic elites and new strategies for extractivism in Chile. European Review of Latin American and Caribbean Studies, 2019, no. 108, pp. 131-152. ISSN 1879-4750. DOI 10.32992/erlacs.10511. Chile, 1990-2019

MANTOVANI, E.T. y SVAMPA, M. En las fronteras del cambio de época. Escenarios de una nueva fase del extractivismo en América Latina. En: K. GABBERT y M. LANG, ¿Cómo se sostiene la vida en América Latina? Feminismos y re-existencias en tiempos de oscuridad [en línea]. Ediciones Abya-Yala. Quito: s.n., 2019.

MOFFAT, K. y ZHANG, A. The paths to social licence to operate: An integrative model explaining community acceptance of mining. Resources Policy, 2014, vol. 39, pp. 61-70. ISSN 0301-4207. DOI 10.1016/j.resourpol.2013.11.003.

OCAMPO-MELGAR, A., SAGARIS, L. y GIRONÁS, J. Experiences of voluntary early participation in Environmental Impact Assessments in Chilean mining. Environmental Impact Assessment Review, 2019, vol. 74, pp. 43-53. ISSN 01959255. DOI 10.1016/j.eiar.2018.09.004.

RICH, J.A.J., MAYKA, L. y MONTERO, A.P.. Introduction The Politics of Participation in Latin America: New Actors and Institutions. Latin American Politics and Society, 2019, vol. 61, no. 2, pp. 1-20. ISSN 1531-426X, 1548-2456. DOI 10.1017/lap.2018.74.

RIOFRANCOS, T.N. Scaling Democracy: Participation and Resource Extraction in Latin America. Perspectives on Politics, 2017 vol. 15, no. 3, pp. 678-696. ISSN 1537-5927, 1541-0986. DOI 10.1017/ S1537592717000901.

SILVA, E., AKCHURIN, M. y BEBBINGTON, A.J. Policy Effects of Resistance against Mega-Projects in Latin America: An Introduction. European Review of Latin American and Caribbean Studies I Revista Europea de Estudios Latinoamericanos y del Caribe, 2018, vol. 0, no. 106, pp. 23. ISSN 1879-4750, 0924-0608. DOI 10.32992/erlacs.10397.

SVAMPA, M. Extractivismo neodesarrollista y movimientos sociales. ¿Un giro ecoterritorial hacia nuevas alternativas? En: GRUPO PERMANENTE DE TRABAJO SOBRE ALTERNATIVAS AL DESARROLLO (ed.), Más allá del desarrollo. Quito: Abya Yala, pp. 185-218, 2012. 
